Check for updates

Cite this: RSC Adv., 2018, 8, 33291

Received 23rd August 2018

Accepted 20th September 2018

DOI: $10.1039 / \mathrm{c} 8 \mathrm{ra07067a}$

rsc.li/rsc-advances

\section{Alloying copper and palladium nanoparticles by pulsed laser irradiation of colloids suspended in ethanol}

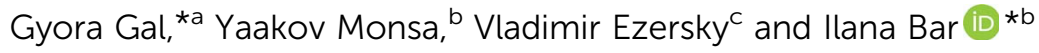

Nanoparticles (NPs) of copper, palladium and $\mathrm{Cu}_{0.8} \mathrm{Pd}_{0.2}$ alloy have been prepared by pulsed laser ablation/ irradiation in ethanol, by the second harmonic of a pulsed Nd: YAG laser $(532 \mathrm{~nm}, \sim 5 \mathrm{~ns}, 10 \mathrm{~Hz}$ ). The monometallic NPs were synthesized by laser ablation of pure bulk targets immersed in ethanol and the alloyed ones by laser irradiation of stirred mixtures of suspended monometallic colloids. The suspensions were irradiated through two distinctive configurations, including lateral collimated and top focused beams that reached the corresponding fluences for NPs vaporization and for extensive plasma formation. The generated NPs were characterized by ultraviolet-visible absorption spectrometry, low and high-resolution transmission electron microscopy, energy-dispersive spectroscopy and selected area electron diffraction. The first fluence regime afforded the synthesis of alloyed NPs in the few $\mathrm{nm}$ diameter range, where alloying was somewhat disturbed by agglomeration, while the second led to larger size NPs and faster alloying, due to laser scattering by the plasma. These findings were supported and interpreted by the particle heating-melting-evaporation model. The approach developed here, assisted by the model and the various characterization methods, proved to control the alloying process and the size distribution of the NPs and to give the best indication for its progress.
\end{abstract}

\section{Introduction}

Synthesis of nanoparticles (NPs) by pulsed laser ablation/ irradiation in liquids (PLAL/PLIL) is a very promising approach and attracts considerable interest, due to the important role that NPs may play in different fields, as well as the expected ramp in their future use. ${ }^{1}$ The physicochemical properties of the NPs are mainly affected by their size, crystalline structure, composition, form and the used solvent. Therefore, investigations are motivated by the possibility of controlling these features, improving the productivity and obtaining an understanding of the properties and performance of the NPs. In PLAL, a pulsed-laser is focused on a solid target immersed in liquid, leading to evaporation of the material, which turns into an expanding plasma plume, confined by the liquid. PLIL is related to irradiation of colloids suspended in liquids ${ }^{2,3}$ where the energy is selectively absorbed by the particles, leading to their heating without significant thermal energy transfer to the liquid. The temperatures and phase changes, reached by the NPs, mainly depend on laser fluences, and NP size and optical

${ }^{a}$ Department of Chemistry, Nuclear Research Center Negev, P.O. Box 9001, Beer-Sheva 8419001, Israel.E-mail: gyorag@gmail.com

${ }^{b}$ Department of Physics, Ben-Gurion University of the Negev, Beer-Sheva 8410501, Israel. E-mail: ibar@bgu.ac.il

'Ilse Katz Institute of Nanoscale Science and Technology, Ben-Gurion University of the Negev, Beer-Sheva 8410501, Israel and thermodynamic properties, as well as the index of refraction of the liquid. In the dwell time between pulses, the particles completely cool down to their original temperature and recrystallize. Besides particle reshaping, this method can be used for NP alloying, by irradiation of mixed colloidal suspensions. ${ }^{3-8}$

NPs, containing more than a single metallic element, may contribute synergistic effects for different applications. Some examples include catalytic CO oxidation, ${ }^{9}$ photocatalytic hydrogen production, ${ }^{10}$ fuel cell anodes, ${ }^{11}$ reduction of 4-nitrophenol, ${ }^{12}$ electrocatalytic oxygen reduction in acidic medium, ${ }^{13}$ electrocatalytic methanol oxidation, ${ }^{14}$ and others. ${ }^{15-17}$ It is anticipated that alloyed palladium $(\mathrm{Pd})$ and copper $(\mathrm{Cu})$ NPs may be exceptionally cooperative for promoting hydrogenation reactions, with supported $\mathrm{Cu}$ being the catalyst of choice. ${ }^{18-23}$ This is due to the very low activation energy and adsorption energy of Pd for dissociative adsorption of hydrogen molecules from gas phase, $\sim 10-20$ and $\sim 35-75 \mathrm{~kJ} \mathrm{~mol}^{-1}\left(\mathrm{H}_{2}\right)$, respectively. ${ }^{24,25}$ Dissociated hydrogen atoms, adsorbed by the Pd in the alloy, can rapidly migrate to the $\mathrm{Cu}$, via the spillover mechanism, ${ }^{26,27}$ to greatly enrich the readily active hydrogen on the catalyst and to boost hydrogenation reactions.

Essentially, it is considered that NPs alloying by PLIL, can be very beneficial and a relatively straightforward method for production of catalysts with enhanced activity. Due to the extreme physical disturbances during the fast heating/cooling and crystallization of the vaporized constituents, this method 
can produce very small and defected NPs without surfactants interference. Even NPs with metastable compositions were shown to be easily obtainable by this method. ${ }^{4,5}$ The advantages and benefits offered by these NPs in catalytic applications include high surface to volume ratio, enhanced active sites on the surface due to defects, ${ }^{\mathbf{2 8 - 3 0}}$ diversity of coordination modes of adsorption on different material interfaces, ${ }^{23,30}$ as well as bare surfaces, leading to catalytically active centers.

PLIL can be performed in different fluence regimes, leading to a variety of structures and alloying efficiencies. Particles fragmentation (downsizing) in liquid can occur for laser fluences above those required for their vaporization. ${ }^{31-33}$ Yet, alloying can already be performed at fluences that can bring the particles to their melting temperature. ${ }^{4-7,34}$ Small NPs that undergo melting, by each laser pulse, can grow till the melting temperature cannot be reached anymore, at the same fluence. These NPs may end up as perfect shaped spheres, due to their relative long cooling time in the liquid phase that allows their surface energy relaxation.

In this work, we aimed towards preparation of NPs with best characteristics for catalysis, where the key issues were their high surface to volume ratio, high extent of alloying and high purity. To the best of our knowledge, this is the first attempt where laser induced alloying of $\mathrm{Cu}$ and $\mathrm{Pd}$ was performed. In the first stage, NPs of $\mathrm{Cu}$ and Pd monometals were prepared by PLAL of bulk targets embedded in ethanol and in the second stage, two different configurations of PLIL with collimated, or focused laser beams were applied to mixtures of their colloidal suspensions. The former PLIL strategy reached fluences corresponding to selective NPs vaporization, while the later even higher ones. The first configuration led to very small alloyed $\mathrm{Cu}_{0.8} \mathrm{Pd}_{0.2}$ NPs, while the second supported much faster alloying, at the expense of presence of larger NPs.

\section{Results and discussion}

\section{The experimental concept}

The principles underlying the experimental concept are presented in Figs. 1(a)-(c), showing the different configurations that were employed for PLAL of $\mathrm{Cu}$ and Pd bulk targets and for PLIL of their mixed suspensions. As mentioned above, these configurations are related to different fluence regimes and panels (d) and (e) show the calculated laser fluences for melting and evaporation of NPs of different diameters at $532 \mathrm{~nm}$. The curves are based on the heating-melting-evaporation model, ${ }^{31,35,36}$ accounting for NPs light absorption and thermodynamics of phase transitions. These results were obtained by calculating (see Methods) the energies required for phase change, using the respective thermodynamic data for the monometals and the alloy (melting only, since data for evaporation could not be found) and then dividing these energies by the appropriate absorption cross sections, calculated from Mie theory, ${ }^{37}$ under the assumption that all the absorbed energy was spent on NPs heating, melting and evaporation. Indeed, the resulting curves exhibit the laser fluences required for onset (dashed dot lines) and completion (solid lines) of (d) melting of $\mathrm{Cu}$ (green), $\mathrm{Pd}$ (blue) and $\mathrm{Cu}_{0.8} \mathrm{Pd}_{0.2}$ (cyan) NPs and (e) vaporization of the monometals. These curves correspond to melting and vaporization of NPs of different diameters (dia.), upon start of the processes with each laser pulse of $532 \mathrm{~nm}$ from room temperature. Onset of melting and vaporization corresponds to the phase transition temperature of the monometal NPs, while completion means after supplying the enthalpy required for phase transition. For melting of the alloyed NPs, onset refers to the solidus temperature. It can be clearly seen from Fig. 1(d) and (e) that the melting and evaporation curves of $\mathrm{Cu}$ and Pd NPs differ extensively, while the melting curve for the alloyed NPs is close to that of the $\mathrm{Cu}$ particles. These curves assisted in selecting the laser fluences for irradiation in the two different configurations and also helped in interpreting the measured results.

Considering the selected experimental conditions, the collimated beam [Fig. 1(b)] was adjusted to average fluence of about $0.37 \mathrm{~J} \mathrm{~cm}^{-2}$ at the cuvette entrance, which decreased to $\sim 0.1 \mathrm{~J} \mathrm{~cm}^{-2}$ at its exit, agreeing with the strong absorption by the particles observed in the snapshot. These range of fluences is marked by the cyan color in panel (e), suggesting that in this configuration vaporization and NPs fragmentation could be assessed along the beam path.

For top irradiation, shown in Fig. 1(c), the directed beam was focused into a point half way inside the cuvette with a $2 \mathrm{~J} \mathrm{~cm}^{-2}$ fluence at the liquid-air interface. This fluence is much higher than that required for vaporization and indeed, as can be seen from the snapshot of Fig. 1(c), a bright spot, highly extending out of the beam path, appears just below the gas-liquid interface. The extension of the spot out of the beam path indicates that its origin is probably not from beam scattering by the particles, but rather due to extensive plasma formation. Since the laser fluence was checked to be about 30\% below the breakdown point of the liquid, it is reasonable to assume that the plasma originates from particles breakdown. This plasma highly scatters the laser beam to the spot vicinity, where it is absorbed by the NPs. This region is seen as blurry green secondary scattering, implying much lower fluence there than in the direct beam, passing through the colloidal dispersion. Consequently, it is very possible that a large volume of the colloidal suspension experienced irradiation by a reduced laser fluence. As will be shown below, this irradiation mode led, at least in part, to lower "effective" fluences, mostly corresponding to the melting regime, Fig. 1(d).

The production of the $\mathrm{Cu}$ and Pd NPs was performed by PLAL [Fig. 1(a)] of each of the target metals, while of the $\mathrm{Cu}_{0.8} \mathrm{Pd}_{0.2}$ alloyed NPs by PLIL, through collimated [Fig. 1(b)] and focused [Fig. 1(c)] irradiation of the mixed colloidal dispersions of the monometals for different time durations. The colloidal dispersions contained molar fractions of $80 \pm$ $10 \%$ of $\mathrm{Cu}$ and $20 \pm 10 \%$ of $\mathrm{Pd}$. The large uncertainty is due to the minute weight that could be ablated from the metal targets, before the concentration of the NPs led to absorption of the irradiating laser beam, preventing their efficient production. The resulting NPs were characterized by various methods, including ultraviolet-visible (UV-Vis) absorption spectroscopy and high-resolution electron microscopy techniques. 


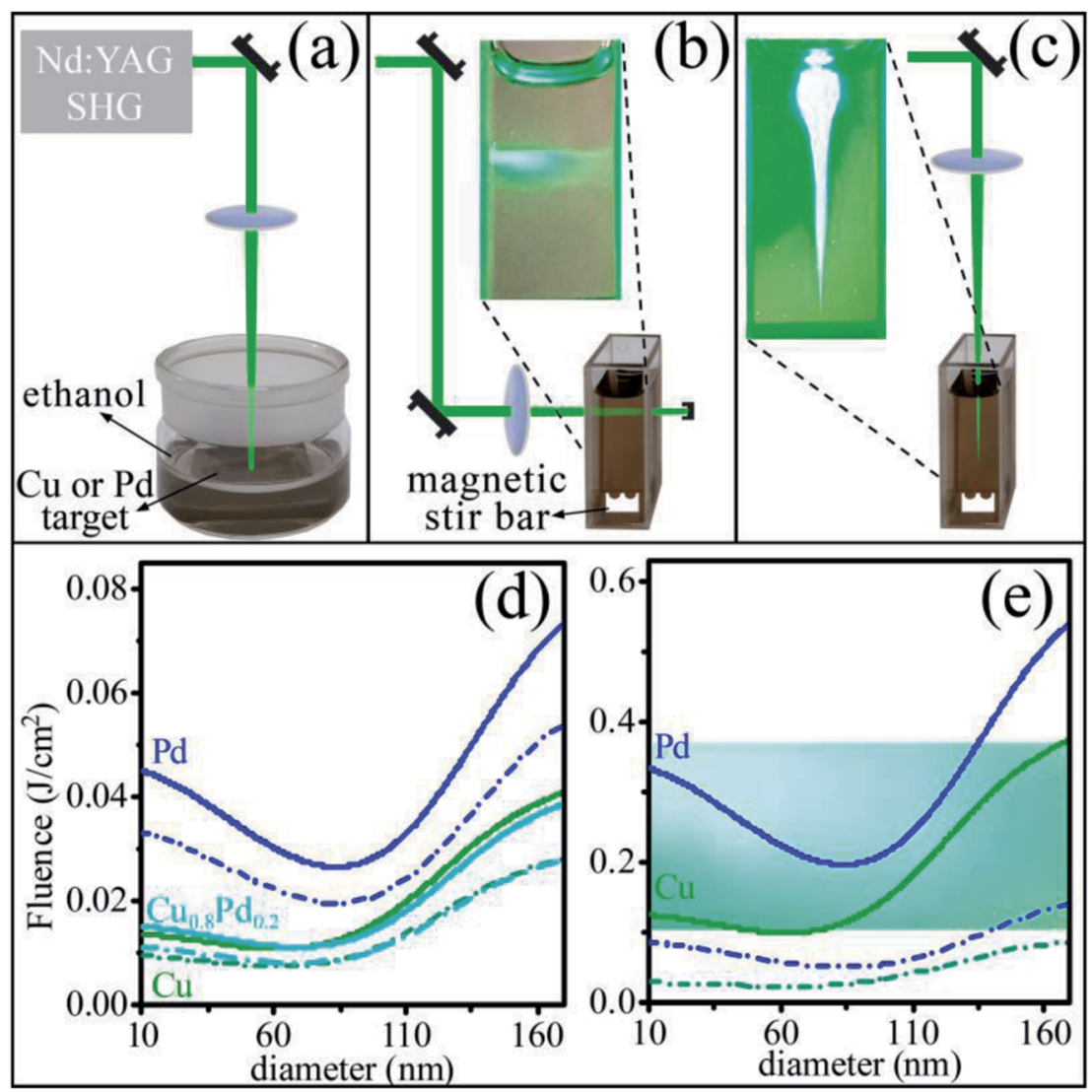

Fig. 1 Schematic experimental set up for nanoparticles (NPs) production from (a) metal bulks and the configurations used for alloying from mixtures of colloidal suspensions with (b) lateral irradiation by a collimated beam and with (c) top irradiation by a focused beam; calculated laser fluences as a function of NPs diameter for a laser wavelength of $532 \mathrm{~nm}$, based on the particle heating-melting-evaporation model, $31,35,36$ required for onset (dashed dot lines) and completion (solid lines) of (d) melting of $\mathrm{Cu}$ (green), $\mathrm{Pd}$ (blue) and $\mathrm{Cu}_{0.8} \mathrm{Pd}_{0.2}$ (cyan) particles and (e) vaporization of the monometal NPs. The snapshots in (b) and (c) show enlarged portions of the beams, passing through the mixed colloidal suspensions. The cyan region in (e) marks the fluence regime attained by the collimated irradiation, shown in (b).

\section{Characterization of the resulting nanoparticles}

The resulting UV-Vis absorption spectra, in the $200-800 \mathrm{~nm}$ range, for each of the suspensions was measured before and shortly after irradiation, see Fig. 2. In particular, the spectrum of the resulting NPs, shown in (a), was obtained following a 1 min stirring of the mixture of $\mathrm{Cu}$ and $\mathrm{Pd}$ suspensions, separately prepared by PLAL. The irradiation times with collimated beam [Fig. 1(b)] were $t_{\text {irad }}=5,60$ and $120 \mathrm{~min}$ and with focused beam [Fig. 1(c)] were $t_{\text {irad }}=1$ and $15 \mathrm{~min}$, and the resultant spectra are shown in (b), (c), (d), (e) and (f) of Fig. 2, respectively.

It is evident that these spectra are characterized by broad absorption bands, where (a)-(c) exhibit features corresponding to a surface plasmon resonance (SPR), at about $580 \mathrm{~nm}$, confirming the presence of metallic Cu NPs. ${ }^{38}$ As can be seen, this peak decreased for longer collimated irradiation [Fig. 1(b)] time, till its vanishing at $t_{\text {irad }}=120 \mathrm{~min}$ in Fig. 2(d). In contrast to this, for focused irradiation, Fig. 1(c), the $\mathrm{Cu}$ SPR peak disappeared already after $1 \mathrm{~min}$, Fig. 2(e), indicating the loss of the $\mathrm{Cu}$ NPs and probably pointing to faster alloying.

The transmission electron microscopy (TEM) images, Fig. 3, allow to assess the morphology and size distribution of the formed NPs. The panels notation here and in the following figures, is adopted from Fig. 2 and the presented data refers to dried samples on TEM grids, prepared from the same suspensions. Fig. 3(a), related to the sample from the $1 \mathrm{~min}$ stirred mixture of $\mathrm{Cu}$ and Pd NPs suspensions, shows that most NPs are small (less than $10 \mathrm{~nm}$ ) and agglomerated. From panels (b), (c) and (d) of Fig. 3 it can be inferred that most NPs from the mixed and stirred suspensions, following 5, 60 and $120 \mathrm{~min}$ of collimated irradiation [Fig. 1(b)], respectively, seem to be mostly small. This is probably due to fragmentation, occurring by the evaporating fluence, although several larger particles also appear as a result of irradiation with the Gaussian beam, which provides low fluences corresponding to melting, in the outer portion of the beam. Hence, under this fluence, the small size of most NPs and the extent of agglomeration could be maintained. This is not the case for panels (e) and (f), which show NPs from mixed and stirred suspensions, irradiated for much shorter times, i.e., $t_{\text {irad }}=1$ and $15 \mathrm{~min}$, respectively, under focused irradiation [Fig. 1(c)]. In this case, most material seems to be in large (less, or above $100 \mathrm{~nm}$ ) spheres, although small and even very tiny NPs can also be observed. As mentioned above, the generation of the large spherical NPs could be attributed to the 


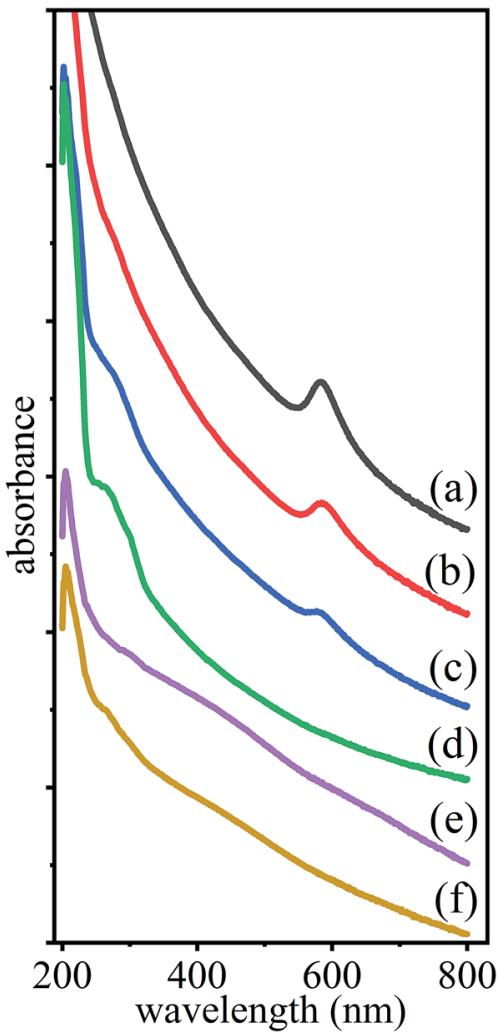

Fig. 2 Absorption spectra of mixtures of suspensions of $\mathrm{Cu}$ and $\mathrm{Pd}(\mathrm{a})$ prepared by pulsed laser ablation in ethanol and then subsequently irradiated by a collimated beam [Fig. 1(b)] for (b) 5, (c) 60 and (d) $120 \mathrm{~min}$, as well as by a focused beam [Fig. 1(c)] for (e) 1 and (f) $15 \mathrm{~min}$.

highly scattered laser beam, which could irradiate significant portions of the suspension, out of the direct beam path, with fluences corresponding to particles melting leading to their size growth. ${ }^{39}$ On the other hand, suspension portions irradiated by the high fluence in the direct beam, preceded toward particles fragmentation and consequently to tiny NPs. The spherical shape of the large NPs is attributed to surface tension during melting and leads to small ratio of contact area to inertia, probably contributing to the loss of agglomeration. ${ }^{40}$

Besides the above characterization by TEM, it could also provide spectroscopic information that might validate the identity of the elements present in the NPs, as well as their concentrations and spatial distributions. This could be obtained from the elemental mapping, shown in Fig. 4, through energy-dispersive spectroscopy (EDS). In particular, panel (a), shows the distribution of each element in a different color, i.e., $\mathrm{Cu}$ (blue) and Pd (green), for NPs obtained from their mixed suspensions, as prepared by PLAL. By performing a color overlap analysis of the two, for the samples prepared from the irradiated mixtures, the appearance of cyan color is observed, either due to overlap of monometallic NPs, or formation of $\mathrm{Cu}_{0.8} \mathrm{Pd}_{0.2}$ alloyed NPs. It can be clearly seen that the extent of cyan color increases as collimated irradiation [Fig. 1(b)] is applied for longer times, i.e., irradiated mixture for $120 \mathrm{~min}$, Fig. 4(d). However, it is obvious that the appearance of cyan in focused irradiation [Fig. 1(c)] of even $1 \mathrm{~min}$ is more extensive,
Fig. 4(e), and it raised upon 15 min irradiation, Fig. 4(f). In addition, it is interesting to note that some of the EDS maps of particles obtained from the mixed suspensions show separated agglomerates of $\mathrm{Cu}$ and Pd NPs [Fig. 4(a)] and to some extent, even following mixing and collimated irradiation of up to $t_{\text {irad }}=$ 60 min, Fig. 4(c).

In addition, to confirm that alloying is indeed responsible for cyan color appearance in the EDS maps, selected area electron diffractions (SAEDs) in TEM were measured and analyzed to obtain lattice parameters. Actually, X-ray diffraction (XRD) measurements would be preferred for this task since they are more accurate, but the amount of NPs was far below that needed for any form of this kind of measurement (except for preirradiated colloidal mixture, where microfocused XRD could be applied, see below). The results for the generated NPs in the individual samples are presented in Fig. 5. Each panel contains an image of the diffraction intensity in the $\mathbf{k}$ vector plane, while superimposed on it and shown in the bottom part of the panels are the background subtracted profiles in white and black, respectively, deduced by the PASAD-tools software. ${ }^{41}$ These profiles were obtained from the SAED ring patterns, by azimuthal integration of their intensities. The resulting profiles were fitted to multiple Voigt peaks with the Origin software, ${ }^{42}$ while accounting for the tabulated peak centers of possible ingredients, ${ }^{43}$ including $\mathrm{Cu}$ (blue), Pd (green), alloyed $\mathrm{Cu} / \mathrm{Pd}$ (cyan) and copper oxides $\left[\mathrm{Cu}_{2} \mathrm{O}\right.$ (orange) and $\mathrm{CuO}$ (violet)].

It should be pointed out that substantial efforts were devoted applying the fitting procedure and revealing the diffractions corresponding to different crystal structures and planes. The difficulty in performing this analysis was due to the broadening of the peaks, forming the profiles, which could be associated with the high interaction between the scattering electrons and the crystal atoms, leading to multiple scatterings of the already reflected electrons. This is considered as a weakness of the technique and diffractions from interaction lengths, exceeding $\sim 50 \mathrm{~nm}$, were estimated to be inadequate for crystallography. ${ }^{44}$ Although this limitation might impact the fit accuracy, it was deduced that this procedure has the potential to qualitatively provide the alloying extent. The peaks related to the alloyed $\mathrm{Cu} /$ Pd NPs, were identified in frame of Vegard's law, ${ }^{45}$ concerning the linear relation, between the molar fraction of the constituents and the alloy lattice parameters.

As can be seen from Fig. 5, the dashed red lines reflecting the sum of the multiple fitted peaks, match well the diffraction profiles, underneath in black. This fit quality could be maintained, by accepting tolerances in peak center values of less than $2 \%$, relative to the tabulated ones. ${ }^{43}$ Substantial existences of $\mathrm{Cu}_{2} \mathrm{O}$ and even some $\mathrm{CuO}$ could be found in the fitted profiles, shown in Fig. 5(a) and (b), corresponding to NPs from the mixed suspension before and following collimated irradiation of $5 \mathrm{~min}$. Essentially, the high peaks of $\mathrm{Cu}$ and Pd indicate no alloying in the former case and only a slight one in the later. In addition, the small shift of the $\mathrm{Cu}$ peak towards that of $\mathrm{Pd}$ in panel (b) could also allude to alloying initiation. Yet, the progress in alloying is best noticed from the fitting of the profiles in Fig. 5(c) and (d), where the colloidal suspensions were irradiated by a collimated beam [Fig. 1(b)] for 60 and $120 \mathrm{~min}$, 

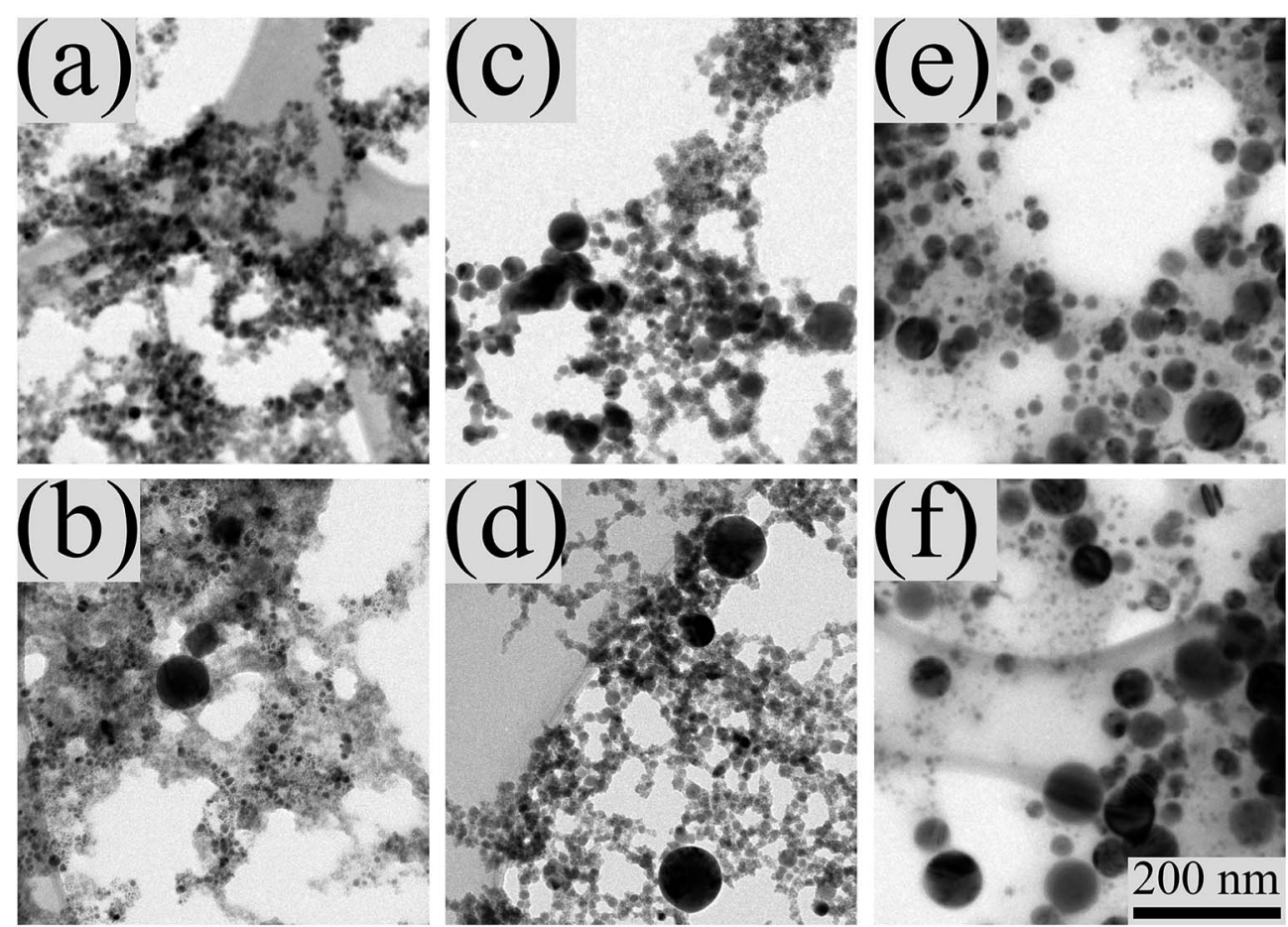

Fig. 3 Transmission electron microscopy images of nanoparticles from mixtures of suspensions of $\mathrm{Cu}$ and Pd (a) prepared by pulsed laser ablation in ethanol (a) and then following subsequent collimated irradiation [Fig. 1(b)] of (b) 120 min, as well as focused irradiation [Fig. 1(c)] of (c) $15 \mathrm{~min}$. The scale bar of $200 \mathrm{~nm}$ at the right bottom corner of (f) is suitable for all the images.

respectively. It is evident that the peaks related to alloyed $\mathrm{Cu} / \mathrm{Pd}$ are most prominent in both cases while the $\mathrm{Cu}, \mathrm{Pd}$ and $\mathrm{Cu}_{2} \mathrm{O}$ peaks were reduced. This is even more pronounced in the fitting of the profiles presented in Fig. 5(e) and (f), which correspond to irradiation by a focused beam [Fig. 1(c)] of the mixed suspensions. It is clearly seen, that for 1 min of focused irradiation, better alloying was obtained, than in collimated irradiation of $120 \mathrm{~min}$. Moreover, $15 \mathrm{~min}$ of focused irradiation led to the
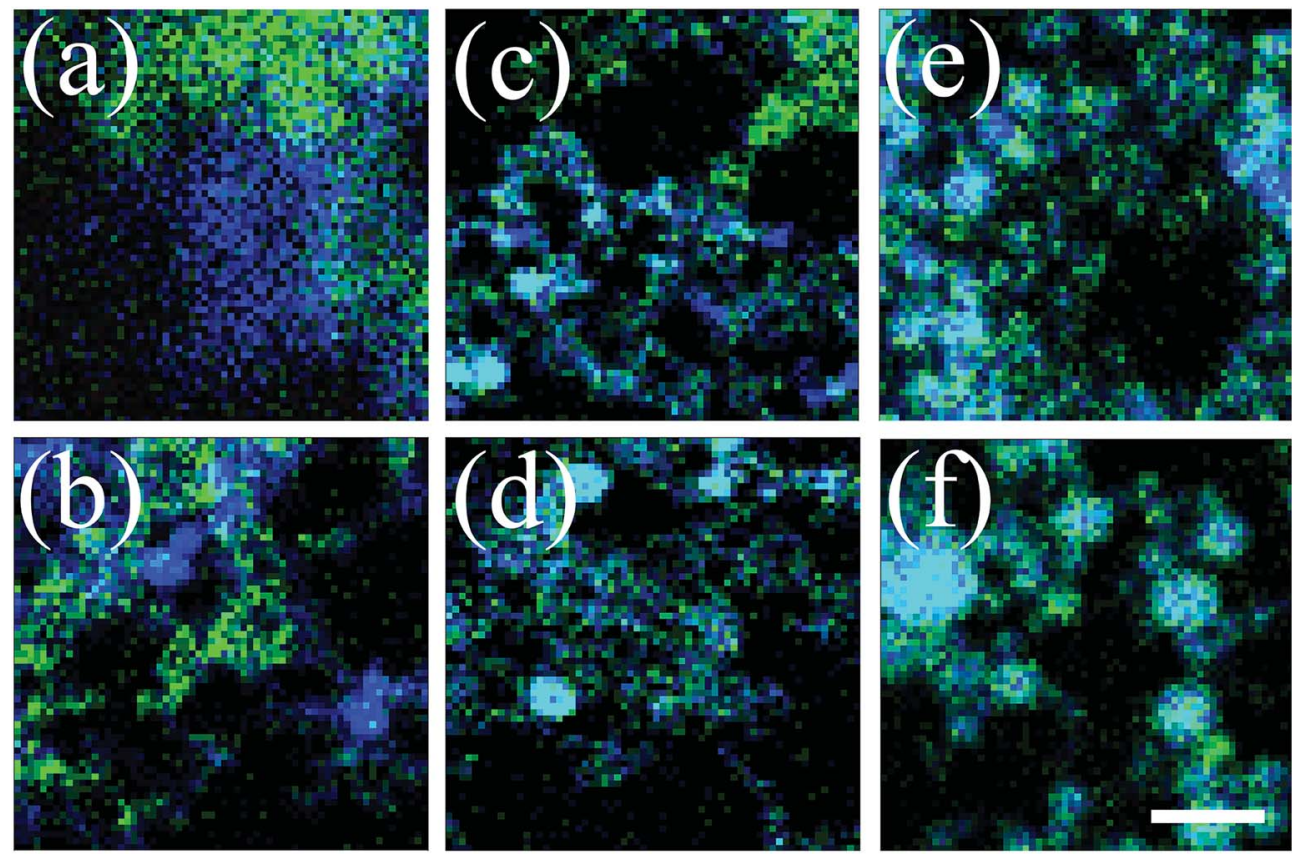

Fig. 4 Energy-dispersive spectroscopy maps of $\mathrm{Cu}$ (blue), $\mathrm{Pd}$ (green) and alloyed $\mathrm{Cu}_{0.8} \mathrm{Pd}_{0.2}$ (cyan) of dried samples of nanoparticles from mixture of suspensions of $\mathrm{Cu}$ and $\mathrm{Pd}$ (a) prepared by pulsed laser ablation in ethanol and then following subsequent collimated irradiation [Fig. 1(b)] of (b) 5, (c) 60 and (d) 120 min, as well as focused irradiation [Fig. 1(c)] of (e) 1 and (f) 15 min. The scale bar at the right bottom corner represents for (a) 20, (b) 250, (c) 300 and for (d), (e) and (f) $200 \mathrm{~nm}$. 

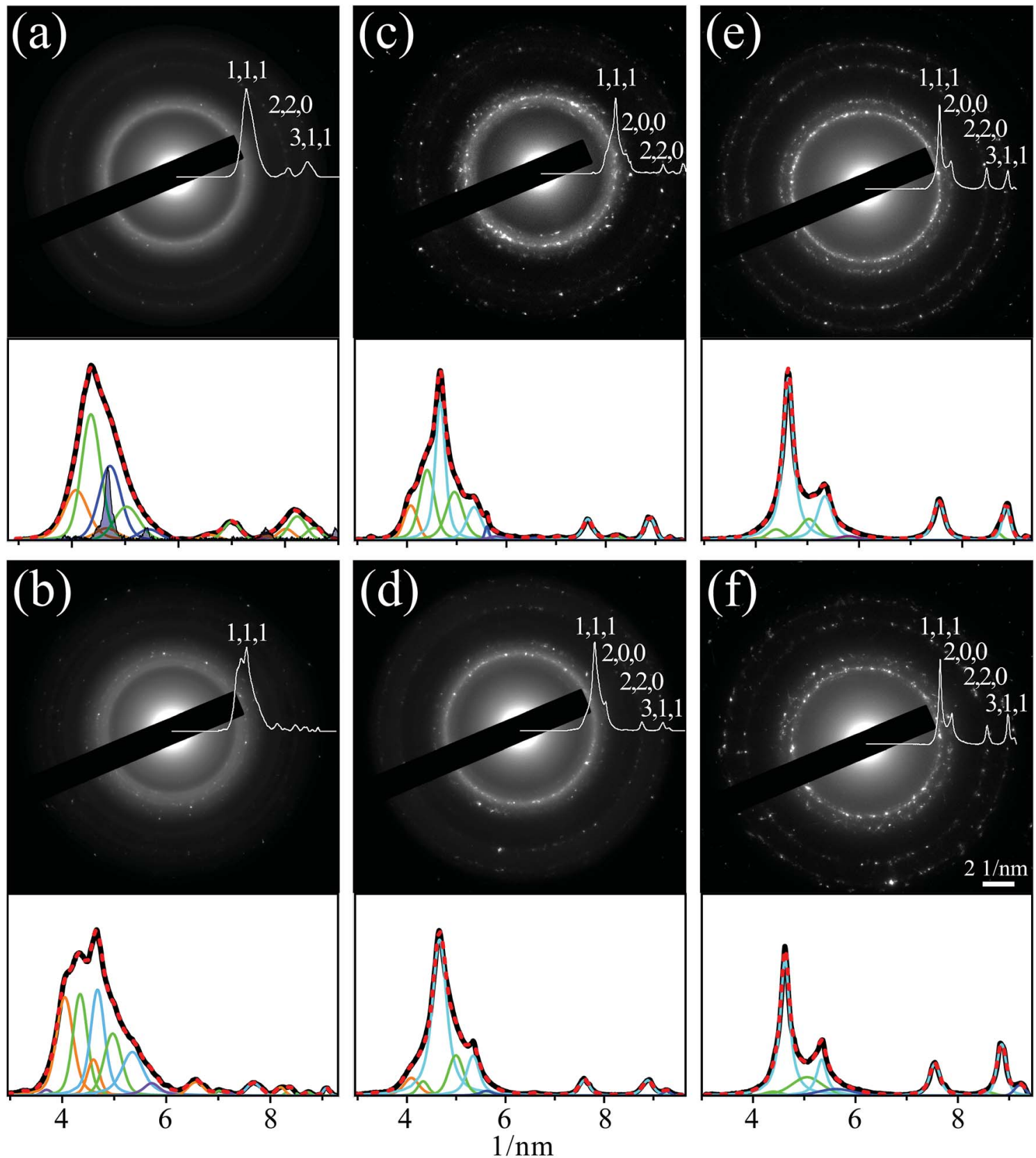

Fig. 5 Electron diffraction patterns and background subtracted profiles of azimuthal integration (panels top) and fits by multiple overlapping Voigt peaks of the latter (panels bottom), corresponding to planes of $\mathrm{Cu}$ (blue), $\mathrm{Pd}$ (green), alloyed $\mathrm{Cu} / \mathrm{Pd}$ (cyan), $\mathrm{Cu} 2 \mathrm{O}$ (orange) and $\mathrm{CuO}$ (violet). The patterns were measured for dried samples of nanoparticles on TEM grids from mixtures of suspensions of Cu and Pd: (a) prepared by pulsed laser ablation in ethanol and then following subsequent collimated irradiation [configuration of Fig. 1(b)] of (b) 5, (c) 60 and (d) 120 min, as well as focused irradiation [Fig. 1(c)] of (e) 1 and (f) 15 min. The darkened peaks in panel (a) were measured by microfocus X-ray diffraction.

sharpest features, corresponding to alloyed $\mathrm{Cu} / \mathrm{Pd}$, and eliminated completely the copper oxides signature, implying that NPs alloying at improved purity could be achieved under these conditions. The results obtained by SAED match very well the indications revealed by the EDS maps, Fig. 4, and the UV-Vis absorption spectra, Fig. 2, confirming that the cyan color in the former is indeed related to alloying and that significantly faster alloying occurred under focused irradiation.

Calculation of the molar fraction of Pd, from the center values of the peaks corresponding to the alloy, leads to estimates of about $35 \%$, which are higher than the $20 \pm 10 \%$, assessed by weighing during suspensions mixing (see Methods). While a simple possible explanation to this discrepancy could be instrumental errors, it is tempting to conjecture that it might be attributed to a $3-5 \%$ molar fraction of oxygen, residing in the lattice as solid solution. This oxygen fraction was calculated from the difference in the face cubic center (fcc) lattice parameters of $\mathrm{Cu}_{2} \mathrm{O}$ and $\mathrm{Cu}$ and can not be considered as a thermodynamically stable structure. Nevertheless, in case of PLIL metastable structures can be obtained, ${ }^{4,5}$ which may lead to this option. 


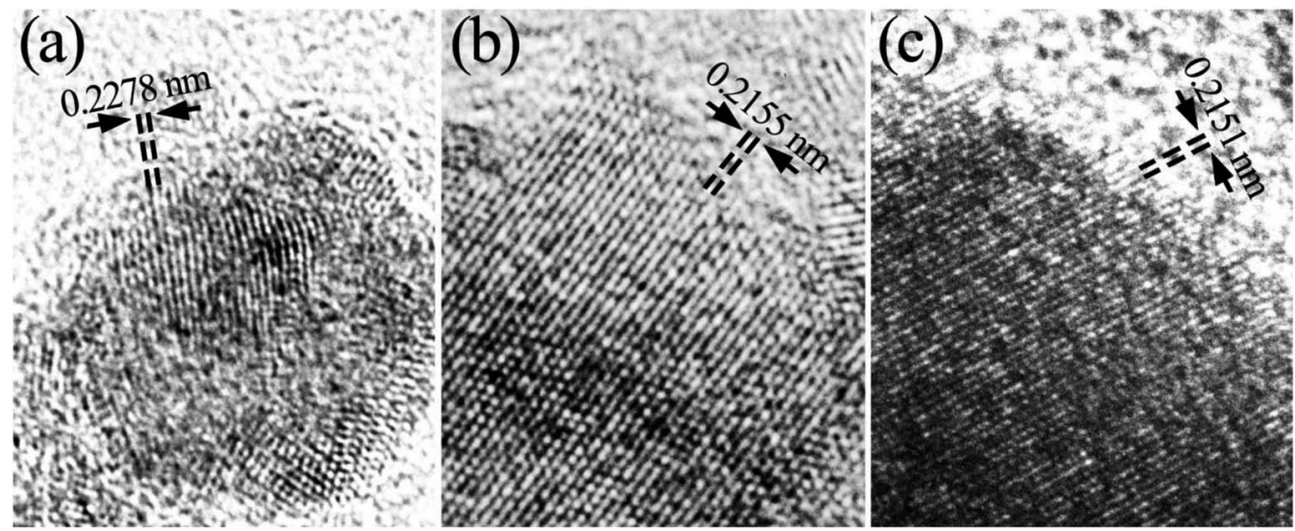

Fig. 6 High resolution transmission electron microscopy images of nanoparticles from mixtures of suspensions of Cu and Pd, prepared by pulsed laser ablation in ethanol (a) and then following subsequent collimated irradiation [Fig. 1(b)] of (b) 120 min, as well as focused irradiation [Fig. 1(c)] of (c) $15 \mathrm{~min}$. Two adjacent $(1,1,1)$ planes of the fcc lattices are marked by parallel lines and the average spacing between the planes was calculated by dividing the total spacing by nineteen (a) thirty four (b) and thirty six (c) spacings.

This assumption is supported by the results of the microfocus XRD measurement, which was performed on minute amounts of dried NPs from the mixed suspension obtained following PLAL. These results are shown in Fig. 5(a) as dark peaks, overlying the SAED profile. Considering the high accuracy expected from the XRD measurement, it was very satisfying to find out that the $\mathrm{Cu}$ peak centers show comparable displacements to those revealed from the fitted profile, supporting the existence of the solid solution with oxygen. It is worth noting that only the peaks related to $\mathrm{Cu}$ could be detected by XRD, probably since the NPs of the other constituents were too small or too thin to be noticeably interacted by the X-rays.

A further assessment of this assumption could be obtained from high resolution TEM (HRTEM) images, taken for the different samples. In Fig. 6, HRTEM micrographs of particular particles from the samples of (a) the preirradiated suspension and of the suspensions following (b) collimated irradiation of $120 \mathrm{~min}$ and (c) focused irradiation of $15 \mathrm{~min}$. In all three particles, the patterns reveal presence of parallel crystallographic planes of crystalline matter. These planes clearly exhibit ordered interplanar spacings. Interplanar spacings of (a) 0.2278 , (b) 0.2151 and (c) $0.2155 \mathrm{~nm}$, marked by parallel lines and arrows in Fig. 6, were obtained by averaging the spacing of nineteen, thirty four and thirty six adjacent planes, respectively. These spacing correspond to the $(1,1,1)$ planes of the fcc lattices of pure palladium and alloyed $\mathrm{Cu} / \mathrm{Pd}$, confirming the formation of these nanocrystals during the PLAL and colloidal mixture irradiation. By using Vegard's law ${ }^{45}$ NPs alloys with compositions of $\mathrm{Cu}_{0.62} \mathrm{Pd}_{0.38}$ (b) and $\mathrm{Cu}_{0.64} \mathrm{Pd}_{0.36}$ (c) were obtained. These findings match the SAED results and support the assumption of $3-5 \%$ molar fraction of oxygen, residing in the alloyed lattice as a solid solution.

An interesting question is why the alloying process is so slow upon irradiation by the collimated laser beam [Fig. 1(b)] and at least two orders of magnitude faster in the case of the focused laser beam [Fig. 1(c)]. It seems that this can not be explained by the high differences in the volumes of the directly irradiated portions of the suspensions, since the irradiated volume is a cylinder of about $3 \mathrm{~mm}$ dia. and $10 \mathrm{~mm}$ length in the former case and a cone with a $2 \mathrm{~mm}$ base dia. and $20 \mathrm{~mm}$ height in the latter case.

An option to refer to this issue is to consider the EDS map of Fig. 4(a) and the TEM image of Fig. 3(a), with other unpresented TEM images of the separate constituents, showing that the NPs are already agglomerated before mixing and reside as separated $\mathrm{Cu}$ and Pd agglomerates, even after 1 min steering of the mixed suspensions. Moreover, large unmixed portions of agglomerates appear even after 60 min mixing and stirring in lateral irradiation [Fig. 4(c)]. One reason could be that significant van der Waals forces control the interaction between the NPs in the agglomerates, probably withstanding the shear stresses exerted by the induced vortexes in the liquid upon steering. Hence, without the presence of these forces almost instantaneous mixing would be expected. It is suggested that plasma ejection in focused irradiation is much more energetic than vapors ejection in collimated irradiation, as can be deduced from the highly extended bright scattering spot, out of the laser beam path, Fig. 1(c). The high kinetic energy of the plasma probably causes mixing of the NPs on much larger volume scales than the ejected vapors. Therefore, the focused irradiation, allows the different constituents in close agglomerates to mix very efficiently with each other, leading to much faster alloying.

\section{Summary}

The application of PLAL to bulk metal targets provided the opportunity to prepare colloidal suspensions of $\mathrm{Cu}$ and Pd NPs of few nm size. Stirred mixtures of the monometallic NPs suspensions were then subjected to various durations of lateral collimated and top focused PLIL, related to completely different fluence regimes. By using different analysis methods, it was possible to reveal the alloying efficiency of the NPs, the extent of oxides purification, and the size distribution, under different preparation conditions. The focused irradiation with fluence affording plasma creation was found to be much more efficient in alloying and in inducing oxides purification but resulted in 
larger size distribution of the alloyed NPs. This drawback can be easily overcome by properly combining these processes, namely using the more efficient process, i.e., focused irradiation for a short time and then collimated irradiation that will lead to fast fragmentation of the already alloyed NPs. The efficient alloying of $\mathrm{Cu}$ and $\mathrm{Pd}$ at the nanoscale and the possibility to control its extent are of most significance. It can be foreseen that the approach used here will contribute to the development of new efficient catalysts, based on the synergetic characteristics of the two metals and the ability to alloy them by PLIL, as particles with bare surfaces and free of surfactants and oxides.

\section{Methods}

\section{Preparation of $\mathrm{Cu}, \mathrm{Pd}$ and their alloyed nanoparticles}

The laser beam used in this work, for both PLAL and PLIL, was the second harmonic (532 nm) of an Nd-YAG laser (Continuum, Surelite SLI-10) with $\sim 5 \mathrm{~ns}$ pulse duration and operating at a $10 \mathrm{~Hz}$ repetition rate. The metal targets for PLAL were of $1 \mathrm{~mm}$ thickness $\mathrm{Cu}$ (99.999\% purity) and Pd (99.8\% purity) and were embedded in clean dehydrated ethanol, 3-4 mm height above the target in an open glass container. A $150 \mathrm{~mm}$ focal length (f.l.) planoconvex lens focused the laser beam to a point beyond the target, so that a $\sim 2 \mathrm{~mm}$ dia. intersected it. This gave a radially averaged fluence of $\sim 2 \mathrm{~J} \mathrm{~cm}^{-2}$, which was enough to sustain the ablation till the NPs concentration in the ethanol grew to a few tens of $\mu \mathrm{g} \mathrm{cm}^{-3}$ in tens of min. The dried metal targets were weighed before and after the ablation, to calculate the molar concentration of the NPs in the suspensions. The subtraction of the latter mass from the former, combined with the small net of NPs yields, led to high uncertainty in the calculated concentrations.

The suspensions of the $\mathrm{Cu}$ and $\mathrm{Pd}$ were mixed to a molar fraction of $20 \pm 10 \%$ of the latter. This mixture suspension was divided to some portions that were inserted in standard $4 \mathrm{~cm}^{3}$ quartz cuvettes for irradiation. For collimated irradiation [Fig. 1(b)] the laser beam was focused by a $250 \mathrm{~mm}$ f.l. planoconvex lens to a point beyond the cuvette, entering through the cuvette side wall, following a $10 \mathrm{~mm}$ path along the suspension and exiting the opposite wall. Due to the long focus and the appropriate distance of the cuvette from the lens a practically collimated beam of $3 \mathrm{~mm}$ dia. irradiated the suspension. For focused irradiation [Fig. 1(c)] the beam was focused at a depth of $20 \mathrm{~mm}$ below the air/liquid interface with $30 \mathrm{~mm}$ f.l. doubleconvex lens, intersecting the liquid/air interface with a $2 \mathrm{~mm}$ dia. The fluence of the focused beam, at the interface, was $2 \mathrm{~J}$ $\mathrm{cm}^{-2}$ and was kept about $30 \%$ below breakdown of the liquid. A magnetic stir bar was employed at the cuvette bottom to mix the suspension along the irradiation.

\section{Nanoparticles characterization}

A TEM (JEOL, JEM-2100F) integrated with EDS and SAED analytical tools, was utilized to obtain the data presented in Fig. 3-6. To avoid Cu background in the EDS measurements, a beryllium sample holder and sample supports of ultrathin carbon film, supported by a lacey carbon film on a 300 mesh gold grid, (Ted Pella Inc.) were used. For the UV-Vis measurements, Fig. 2, a UV-Vis spectrophotometer (Thermo Scientific, Genesys 10S) with standard quartz cuvettes, containing the suspension samples and the clean dehydrated ethanol for background measurements, were employed. Background was automatically subtracted from all measurements.

The XRD measurement, presented in Fig. 5(a), was collected by a system of Bruker, equipped with $\mathrm{I} \mu \mathrm{S} 3.0^{\mathrm{TM}}$ microfocus X-ray source with a molybdenum anode. For sample preparation from the minute amount of powder that could be recovered from the suspension, a $100 \mu \mathrm{m}$ deep hole with a diameter of $110 \mu \mathrm{m}$, drilled in an amorphous bulk, was filled with the powder under a microscope and targeted by the X-ray beam.

\section{Calculations based on the particle heating-melting- evaporation model}

In the calculations presented in Fig. 1(d) and (e), the crosssections for laser absorption as a function of NPs diameter were calculated with the aid of the free Mie-Plot code, ${ }^{46,50}$ using the complex refraction indexes at $532 \mathrm{~nm}$ for $\mathrm{Cu},{ }^{47} \mathrm{Pd}^{48}$ and ethanol. ${ }^{49}$ For the alloyed $\mathrm{Cu}_{0.8} \mathrm{Pd}_{0.2}$ we used $80 \%$ of the complex refraction index of $\mathrm{Cu}$ together with $20 \%$ of that of $\mathrm{Pd}$ (following ref. 51). For the thermodynamic calculations for the monometals, the data tabulated by Pyatenko et al. ${ }^{36}$ was used. For the alloyed $\mathrm{Cu}_{0.8} \mathrm{Pd}_{0.2}$, the solidus temperature was obtained from ref. 52, the molar heat capacity, $C_{\mathrm{p}}$, was calculated from the data of ref. 36 using the same method as for the refraction index and the enthalpy of fusion was taken from ref. 53. The energies required for transferring the particles from room temperature to the different phases were calculated from the thermodynamic data and divided by the calculated absorption cross-sections to obtain the required laser fluences. The particles were assumed to be spherical in these calculations. To justify the constant absorption cross-section, used during the entire heating process, it was assumed that due to the fast energy gain the particles reach phase change only after all the energy is absorbed. Furthermore, it was estimated ${ }^{36}$ that any significant heat exchange between the particles and the liquid cannot occur during this short heating time.

\section{Conflicts of interest}

There are no conflicts to declare.

\section{Acknowledgements}

This work was supported by the Pazy foundation, under grant No. 286/18 and the Israel Science Foundation founded by The Israel Academy of Science. The authors also thank Roee Friedman and Mishael Hornik, from the Israel Atomic Energy Commission who conducted the microfocus XRD measurement. 


\section{References}

1 D. Zhang, B. Gökce and S. Barcikowski, Chem. Rev., 2017, 117, 3990-4103.

2 H. Q. Wang, A. Pyatenko, K. Kawaguchi, X. Y. Li, Z. Swiatkowska-Warkocka and N. Koshizaki, Angew. Chem., Int. Ed., 2010, 49, 6361-6364.

3 Z. Swiatkowska-Warkocka, K. Koga, K. Kawaguchi, H. Q. Wang, A. Pyatenko and N. Koshizaki, RSC Adv., 2013, 3, 79-83.

4 Z. Swiatkowska-Warkocka, A. Pyatenko, F. Krok, B. R. Jany and M. Marszalek, Sci. Rep., 2015, 5, 09849.

5 Z. Swiatkowska-Warkocka, A. Pyatenko, K. Koga, K. Kawaguchi, H. Wang and N. Koshizaki, J. Phys. Chem. C, 2017, 121, 8177-8187.

6 Y. Chen and C. Yeh, Chem. Commun., 2001, 371-372.

7 G. C. Messina, M. G. Sinatra, V. Bonanni, R. Brescia, A. Alabastri, F. Pineider, G. Campo, C. Sangregorio, G. LiDestri, G. Sfuncia, G. Marletta, M. Condorelli, R. P. Zaccaria, F. De Angelis and G. Compagnini, J. Phys. Chem. C, 2016, 120, 12810-12818.

8 N. G. Semaltianos, R. Chassagnon, V. Moutarlier, V. Blondeau-Patissier, M. Assoul and G. Monteil, Nanotechnology, 2017, 28, 155703.

9 J.-H. Liu, A.-Q. Wang, Y.-S. Chi, H.-P. Lin and C.-Y. Mou, J. Phys. Chem. B, 2005, 109, 40-43.

10 E. Aronovitch, P. Kalisman, S. Mangel, L. Houben, L. Amirav and M. Bar-Sadan, J. Phys. Chem. Lett., 2015, 6, 3760-3764.

11 X. Ji, K. T. Lee, R. Holden, L. Zhang, J. Zhang, G. A. Botton, M. Couillard and L. F. Nazar, Nat. Chem., 2010, 2, 286-293.

12 J. Zhang, G. Chen, D. Guay, M. Chaker and D. Ma, Nanoscale, 2014, 6, 2125-2130.

13 F. Godınez-Salomon, M. Hallen-Lopez and O. Solorza-Feria, Int. J. Hydrogen Energy, 2012, 37, 14902-14910.

14 G. T. Fu, R. G. Ma, X. Q. Gao, Y. Chen, Y. W. Tang, T. H. Lub and J. M. Lee, Nanoscale, 2014, 6, 12310-12314.

15 H. Zhang, J. Okuni and N. Toshima, J. Colloid Interface Sci., 2011, 354, 131-138.

16 Q. Wan, P. Li, J. Shan, F. Zhai, Z. Li and X. Qu, J. Phys. Chem. C, 2015, 119, 2925-2934.

17 J. J. Plata, J. Graciani, J. Evans, J. A. Rodriguez and J. F. Sanz, ACS Catal., 2016, 6, 4608-4615.

18 Y. H. Wang, W. G. Gao, H. Wang, Y. E. Zheng, W. Na and K. Z. Li, RSC Adv., 2017, 7, 8709-8717.

19 Y. Zhang, Q. Sun, J. Deng, D. Wu and S. Chen, Appl. Catal., A, 1997, 158, 105-120.

20 G. C. Chinchen, P. J. Denny, D. G. Parker, M. S. Spencer and D. A. Nhan, Appl. Catal., 1987, 30, 333-338.

21 Y. Yang, J. Evans, J. A. Rodriguez, M. G. White and P. Liu, Phys. Chem. Chem. Phys., 2010, 12, 9909-9917.

22 J. Yoshihara and C. T. Campbell, J. Catal., 1996, 161, 776782.

23 J. Graciani, K. Mudiyanselage, F. Xu, A. E. Baber, J. Evans, S. D. Senanayake, D. J. Stacchiola, P. Liu, J. Hrbek, J. F. Sanz and J. A. Rodriguez, Science, 2014, 345, 546-550.
24 M. Johansson, E. Skúlason, G. Nielsen, S. Murphy, R. M. Nielsen and I. Chorkendorff, Surf. Sci., 2010, 604, 718-729.

25 J. Roques, C. Lacaze-Dufaure and C. Mijoule, J. Chem. Theory Comput., 2007, 3, 878-884.

26 H. L. Tierney, A. E. Baber, J. R. Kitchin and E. C. H. Sykes, Phys. Rev. Lett., 2009, 103, 246102.

27 M. Ramos, A. E. Martınez and H. F. Busnengo, Phys. Chem. Chem. Phys., 2012, 14, 303-310.

28 M. Behrens, F. Studt, I. Kasatkin, S. Kühl, M. Hävecker, F. Abild-Pedersen, S. Zander, F. Girgsdies, P. Kurr, B. L. Kniep, M. Tovar, R. W. Fischer, J. K. Nørskov and R. Schlögl, Science, 2012, 336, 893-897.

29 A. Migani, G. N. Vayssilov, S. T. Bromley, F. Illas and K. M. Neyman, Chem. Commun., 2010, 46, 5936-5938.

$30 \mathrm{~J} . \quad$ H. K. Pfisterer, Y. Liang, O. Schneider and A. S. Bandarenka, Nature, 2017, 549, 74-77.

31 A. Takami, H. Kurita and S. Koda, J. Phys. Chem. B, 1999, 103, 1226-1232.

32 V. Amendola and M. Meneghetti, J. Mater. Chem., 2007, 17, 4705-4710.

33 F. Hajiesmaeilbaigi, A. Mohammadalipour, J. Sabbaghzadeh, S. Hoseinkhani and H. R. Fallah, Laser Phys. Lett., 2006, 3, 252-256.

34 M. Ganjali, M. Ganjali, S. Khoby and M. A. Meshkot, NanoMicro Lett., 2011, 3, 256-263.

35 A. Pyatenko, M. Yamaguchi and M. Suzuki, J. Phys. Chem. C, 2009, 113, 9078-9085.

36 A. Pyatenko, H. Wang, N. Koshizaki and T. Tsuji, Laser Photonics Rev., 2013, 7, 596-604.

37 G. Mie, Ann. Phys., 1908, 330, 377-445.

38 P. Liu, H. Wang, X. Li, M. Ruib and H. Zeng, RSC Adv., 2015, 5, 79738-79745.

39 A. Pyatenko, H. Wang and N. Koshizaki, J. Phys. Chem. C, 2014, 118, 4495-4500.

40 G. Stroh, in Coagulation and Flocculation: theory and applications. ed. B. Dobias, Surfactant science series, Marcel Dekker, Inc., New York, 1993, vol. 47, p. 656.

41 C. Gammer, C. Mangler, C. Rentenberger and H. P. Karnthaler, Scr. Mater., 2010, 63, 312-315.

42 OriginPro 2016, OriginLab Corporation, https:// www.originlab.com.

43 P. Villars and K. Cenzual, Pearson's Crystal Data - Crystal Structure Database for Inorganic Compounds (on CD-ROM), release 2011/12, ASM International, Materials Park, Ohio, USA.

44 L. B. McCusker and C. Baerlocher, Z. Kristallogr., 2013, 228, 1-10.

45 L. Vegard, Z. Phys., 1921, 5, 17-26.

46 P. Laven, MiePlot v4.6.07, 6 June 2017, https:// www.philiplaven.com/mieplot.htm.

47 P. B. Johnson and R. W. Christy, Phys. Rev. B: Solid State, 1972, 6, 4370-4379.

48 Handbook of Optical Constants of Solids, ed. E. D. Palik, Academic Press, New York, 1985.

49 S. Kedenburg, M. Vieweg, T. Gissibl and H. Giessen, Opt. Mater. Express, 2012, 2, 1588-1611. 
50 S. Hashimoto, D. Werner and T. Uwada, J. Photochem. Photobiol., C, 2012, 13, 28-54.

51 T. Shiraishi and R. J. D. Tilley, Gold Bull., 2014, 47, 75-82.
52 P. R. Subranian and D. E. Loughlin, J. Phase Equilib., 1991, 12, 231-243.

53 J. Davoodi, M. Ahmadi and H. Rafii-Tabar, Mater. Sci. Eng., A, 2010, 527, 4008-4013. 